\title{
Applying Radial Basis Function Networks to Fault Diagnosis of Motorized Spindle
}

\author{
Zhe Li and Kesheng Wang* \\ Department of Production and Quality Engineering \\ Norwegian University of Science and Technology \\ Trondheim, Norway \\ zhe.li@ntnu.no; kesheng.wang@ntnu.no
}

\author{
Jinghui Yang and Yavor Stefanov \\ Department of Industry Engineering \\ Shanghai Polytechnic University \\ Shanghai, China \\ Jinghui.yang@ @spu.edu.cn
}

\begin{abstract}
In a motorized spindle, due to the complexity of the system and nonlinear relationship between features and types of faults, it is difficult and inefficient to use traditional methods or physical models for the fault diagnosis. This paper focuses on the research on applying Radial Basis Function (RBF) Networks for fault detection and classification in the motorized spindle. As a data driven model with high efficiency, RBF networks has the advantage solving the nonlinear problems and dealing with the contradictory samples in the training process. In this research, the data, including rotating speed, temperature, and acceleration signals with three axes $(X, Y$ and $Z)$, are collected from a dynamic balancing platform to evaluate the working condition and detect the potential faults of the motorized spindle.
\end{abstract}

Keywords - motorized spindle; Radial Basis Function; fault diagnosis

\section{INTRODUCTION}

As the core component of $\mathrm{CNC}$ machines, motorized spindle have significant impacts on the dynamic performance of the whole system. An unexpected failure may not only result in decrease of geometric accuracy, but also devastating accidents and financial losses for the company. In addition, early detection of faults can prevent them from growing and eventually turning into serious problems [1]. However, owing to the complicated and nonlinear relationship between the features, extracted from collected data, and types of failures, which may happen in motorized spindle, it is difficult to apply traditional methods or physical model to detect and classify faults in motorized spindle. Therefore, it is necessary to apply computational learning theory for fault diagnosis in motorized spindle.

A number of factors such as, damage on parts, lack of lubrication, extended periods of running at high speeds, incorrect tool path programming, spindle misalignment or even a fatal crash of the machine tool, may cause the failures of spindles. Those failures could results in a poor surface finish, vibration or excessive heat build-up of the spindle [2]. In order to detect and recognize the fault on motorized spindles, this paper focus on the research of applying machine-learning technologies for the diagnosis on motorized spindles.

In a motorized spindle, many signals collected from a variety of sensors may imply the features of certain faults, such as temperature, vibration, energy consumption, acoustic signal, noise signal et al. For example, Lei et al. [3] proposed a thermal error model based on multivariate autoregressive method to forecast the thermal error. In that model, not only the relationship between thermal deformation and its lagged value but also the relationship between thermal deformation and the present and lagged value of temperature rise was counted into consideration. Chang et al. [4] applied a piezo transducer to check and record the condition of spindle rotation through monitoring the vibration signals. Deng et al [5] used envelope spectrum and wavelet analysis to extract the information of fault from the noise signals of spindles for fault detection in machine tools.

In the research field of Artificial Intelligence (AI), a Radial Basis Function (RBF) network is one kind of Artificial Neural Networks (ANNs) that applies radial basis functions as activation functions to map the real condition of the targets through series of inputs. The output of the network is a linear combination of radial basis functions of the inputs and neuron parameters. In ANNs different Radial Basis Functions are utilized in various areas such as approximation process, pattern recognition, optimization etc. [6].

The remaining part of the paper is organized as follows: Section 2 briefly presents the equipment in the experiment and the method to inject failures. Section 3 discusses the principle of RBF networks for fault diagnosis in the motorized spindle. Section 4 shows how we establish the data warehouse, extract features from raw data, and map the failure for the spindle through RBF networks. Conclusions and future research work are summarized in the last section.

\section{EXPERIMENT SET-UP}

In this paper, a dynamic balancing platform is used to simulate the real working condition of a motorized spindle, as shown in Fig. 1. The data of temperature, vibrations at three axes, and rotating speed are collected as the variables in order to monitor the working condition of the spindle. 


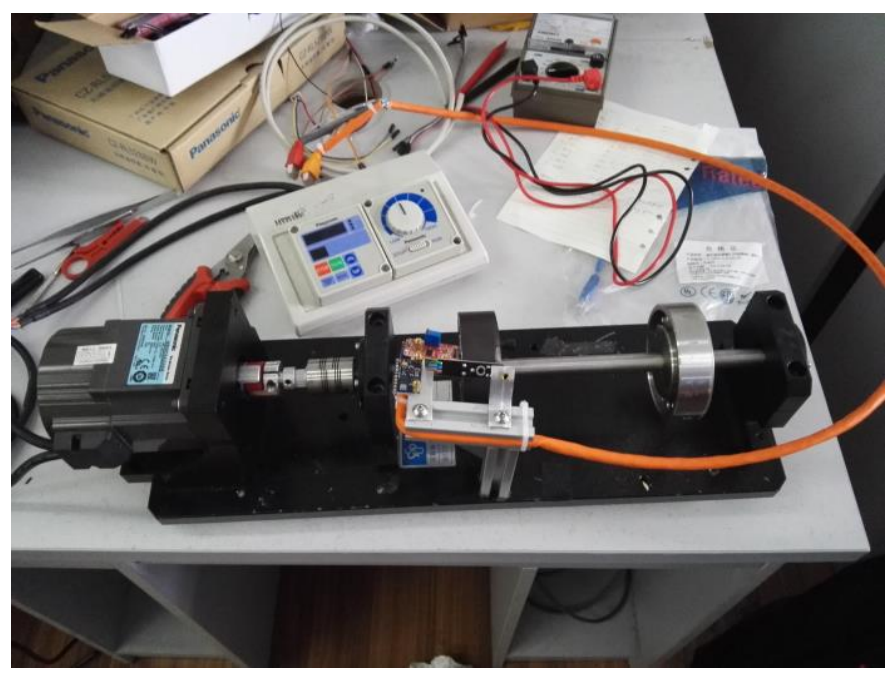

Fig. 1. The set-up of the experiment

In this paper, the main object is the bearing on the spindle as shown in Fig. 2. There is an interference fit between the bearing and main spindle. In the experiment, we will collect the data under both normal and abnormal working condition. During the data collection of abnormal working condition, a new bearing, which has been made some damage in advance (shown in Fig. 3) was installed to simulate the failure on the spindle.

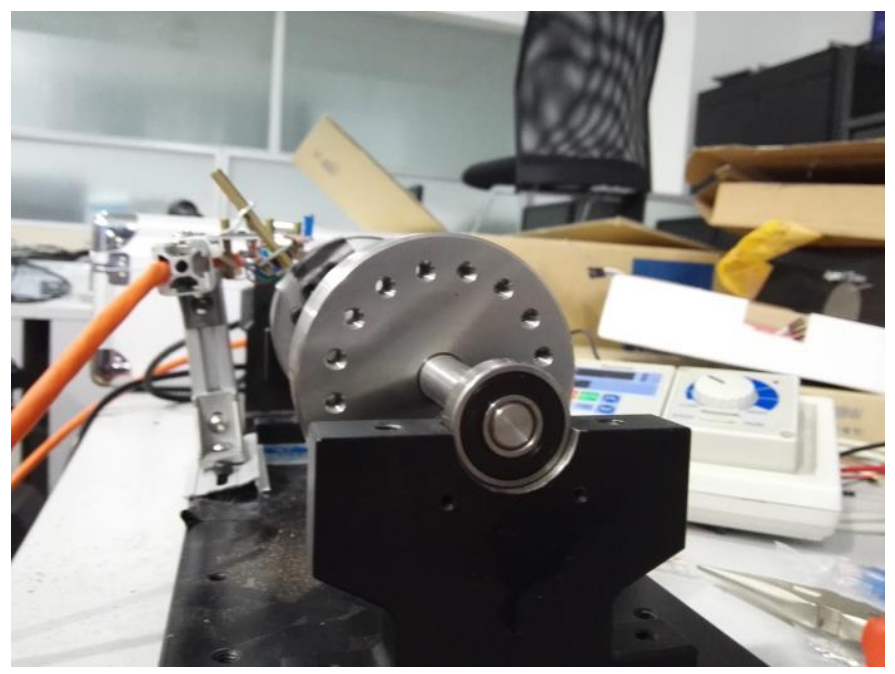

Fig. 2. Interference fit between the bearing and main spindle

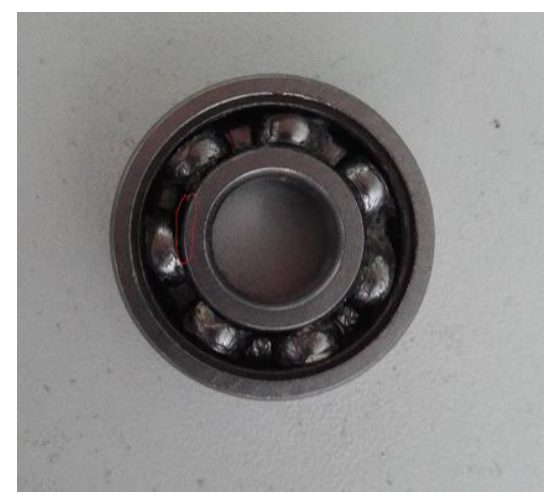

Fig. 3. The damaged bearing with a man-made fault

During the experiment, the data, including temperature, vibration, and rotating speed, will be collected under the normal working condition. After the fault injected, the data under failure will be collected as the abnormal working condition. Both the data under normal and abnormal will be used as the inputs to train a RBF neural network for the fault recognition in the spindle. In next section, the RBF neural networks applied in this paper will be introduced.

\section{RBF NETWORK FOR FAULT DIAGNOSIS}

RBF neural network architecture is of three layers, namely input, hidden and output. As shown in Fig. 4, in the model, inputs are $X_{1}, X_{2}, X_{3}, \ldots, X_{n}$, which present the data collected from the platform. Radial centers are $\mu_{1}, \mu_{2}, \ldots, \mu_{\mathrm{L}}$. In hidden layer $\varphi_{1} ; \varphi_{2} ; \varphi_{3}, \ldots, \varphi_{\mathrm{L}}$ are the radial basis functions for distance between inputs and centers. In addition, the number radial basis functions $\mathrm{L}$ employed in the network should be smaller than $\mathrm{N}$ (input vectors). The choice of L may also be a part of training. The desired output is given by $\mathrm{Y}$ which is calculated by proper selection of $\mathrm{W}_{\mathrm{ij}}$. Here, $\mathrm{W}_{\mathrm{ij}}$ is the weight of $i$ th function in the output $Y_{j}$. Summation of all $\phi_{i} W_{i j}$ is the output of the network.

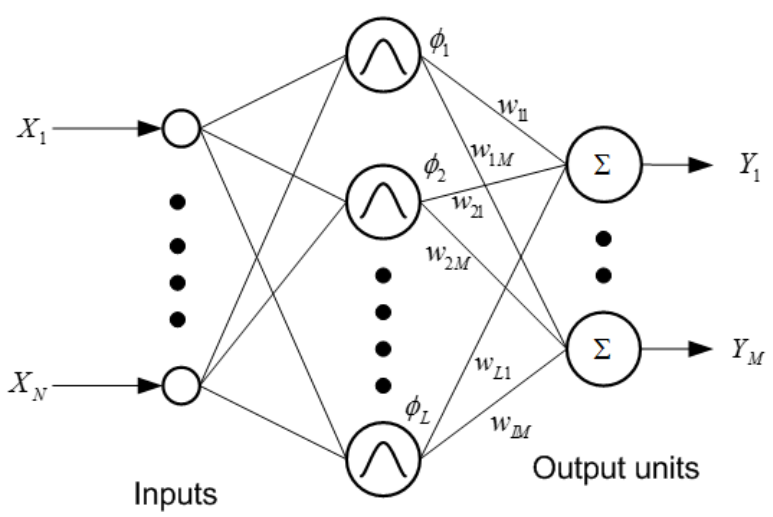

Hidden units

Fig. 4. The structure of Radial Basis Function networks 
Here, Gaussian function is chosen as the radial basis function, see Equation 1. Euclidian distance and maximum distance from centres are $\|x-\mu\|$ and $\sigma$. Therefore, the output of the RBF neural network can be calculated through Equation 2.

$$
\begin{aligned}
& G_{\sigma}(x ; \mu)=\exp \left(-\frac{1}{2 \sigma^{2}}\|x-\mu\|^{2}\right) \\
& F^{*}(x)=\sum_{i=1}^{M} w_{i} \varphi_{i}(x)+w_{o}=\sum_{i=0}^{M} w_{i} \varphi_{i}(x)
\end{aligned}
$$

The training of the RBF network is performed in a supervised manner, i.e., for each training input pattern, a corresponding output pattern (the function to be approximated) is presented. During training, the output layer weights (w), centres of $\operatorname{RBF}(\mu)$ and widths or standard deviation $(\sigma)$ are adapted such that the linear combination of RBF neurons eventually approximates the given function. After training RBF networks through the data both in normal and failure condition, it can map the failures for fault diagnosis in motorized spindles.

\section{EXPERIMENT RESULTS}

Fig. 5 and Fig. 6 show the raw data collected under both normal working condition and the working condition after failure injected, which includes the acceleration signals from three axes and the rotating speed.

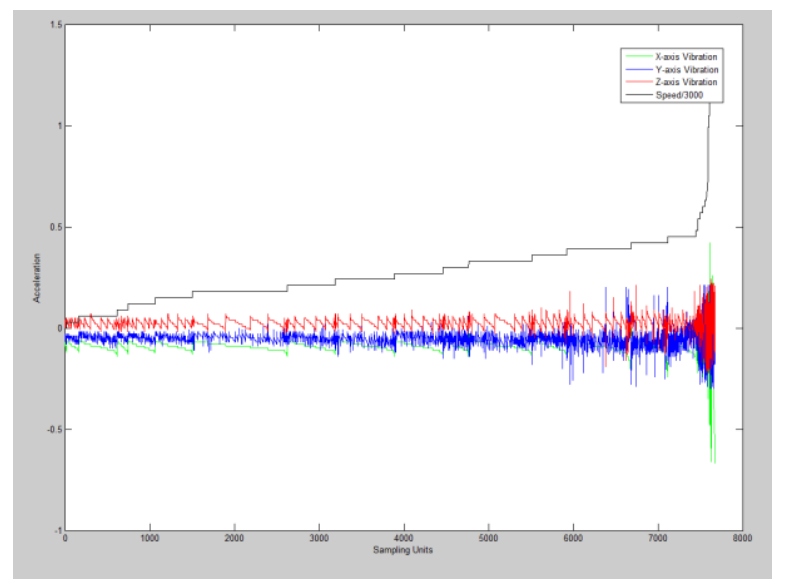

Fig. 5. Raw data collected under normal working condition

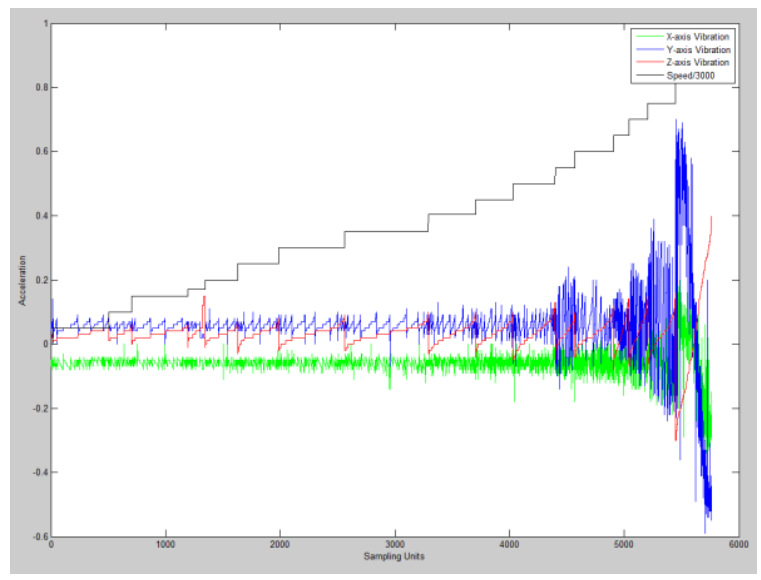

Fig. 6. Raw data collected after the failure injected

The raw data collected from the spindle is stored in the local database directly. In order to extract the feature of the vibrations for RBF training, a data warehouse is established to store the feature information, which can be used to train the RBF networks directly. We extracted the amplitude from the raw data as the inputs for RBF networks, as shown in Fig. 7 and Fig. 8.

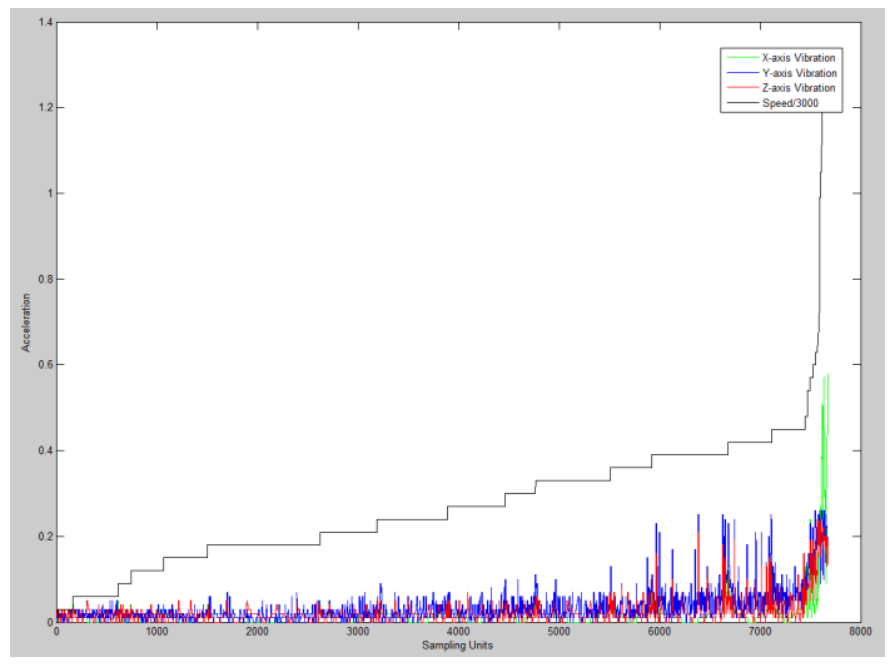

Fig. 7. Feature extracted from normal working condition 


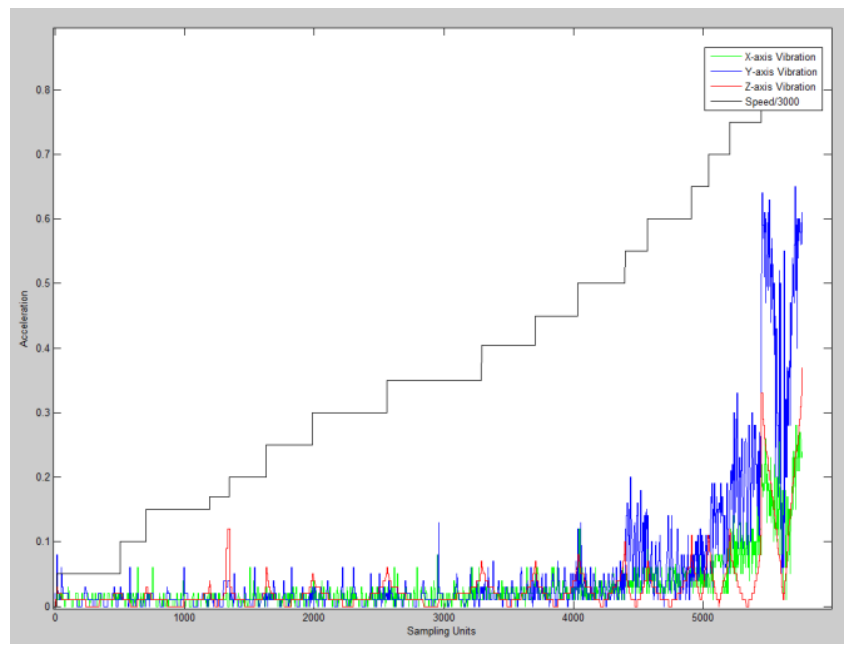

Fig. 8. Feature extracted from failure

A selection of 7673 samples under normal working condition and 5764 samples under failure was applied as the inputs to train the RBF networks. We define the outputs of normal working condition is 0 , while the failure is 1 . During the training process, $70 \%$ of the experiment data was applied to train and adjust the network according to the error between the targets and outputs. The rest remaining 30\% data was divided into two groups: validation and testing. The validation data is used to estimate the condition of the network's generalization, which means the training will cease if the generalization stops improving. And the testing data has no effect on the training process, so an independent assessment can be made according to the testing results. The training result is shown in Fig. 9, the best validation performance is 0.14295 at epoch 95 , which means the average error between the actual and predicted values is 0.14295 .

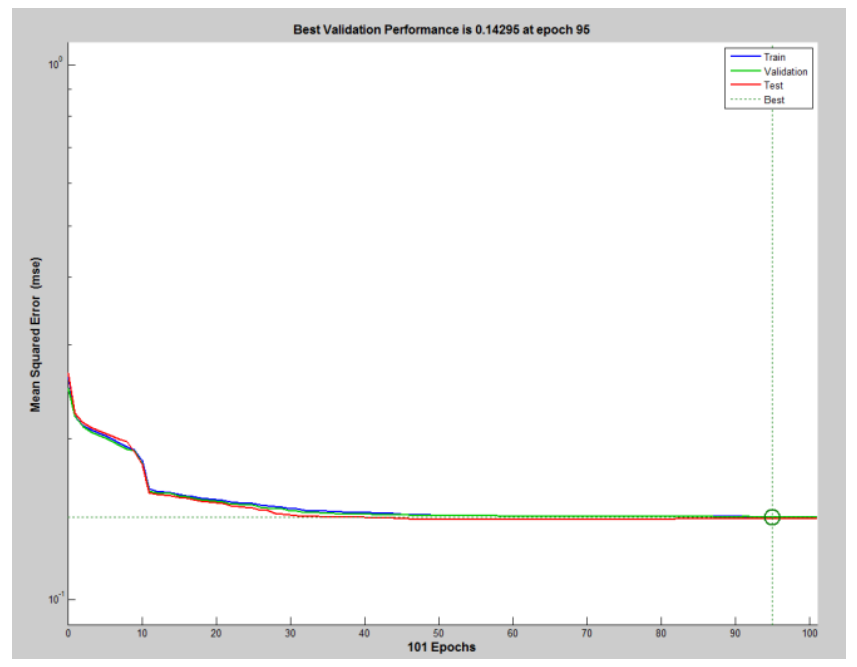

Fig. 9. Training result of RBF network

\section{CONCLUSIONS AND FUTURE WORK}

Motorized spindle is a strong coupled complex system, which causes the difficulty for fault diagnosis and prognosis in motorized spindle. This paper focuses on applying RBF Networks for fault detection and classification in motorized spindle. Compared with traditional back-propagation (BP) neural network, RBF has a better performance in finding global optimum and is more suitable for fault diagnosis, but lacks empirical [7]. Therefore, the authors established the RBF network model to detect and recognize the fault on motorized spindles. As the result shows, the best validation performance is 0.14295 , which means with the help of RBF networks, the accuracy rate for fault diagnosis can reach $85 \%$.

Further research may focus on following aspects:

(1) In this paper, we only inject the failure with damage on the bearings. Further research may focus on increasing the types of injected failures to check whether RBF networks have the ability to recognize failures from several types of faults.

(2) During the experiment, we only extract features and analyse data in time-domain. The methods to extract features from vibration signals may be improved such as applying Wavelet Transform.

\section{REFERENCES}

[1] P. Henriquez, J. B. Alonso, M. A. Ferrer, and C. M. Travieso, "Review of automatic fault diagnosis systems using audio and vibration signals," IEEE Transactions on Systems, Man, and Cybernetics: Systems, vol. 44, pp. 642-652, 2014.

[2] O. Maeda, Y. Cao, and Y. Altintas, "Expert spindle design system," International journal of Machine tools and Manufacture, vol. 45, pp. 537-548, 2005.

[3] L. Chunli and R. Zhiyuan, "Thermal error modeling and forecasting based on multivariate autoregressive model for motorized spindle," Mechanical Science and Technology for Aerospace Engineering, vol. 9, p. 029, 2012.

[4] C.-F. Chang and J.-J. Chen, "Vibration monitoring of motorized spindles using spectral analysis techniques," Mechatronics, vol. 19, pp. 726-734, 2009.

[5] S. Deng, X. Xu, J. Zhang, and X. ZHANG, "Spindle Fault Diagnosis Based on Wavelet and Envelope Analysis," Mach. Tool Hydraul, vol. 37, pp. 219-221, 2009.

[6] A. Paul, P. Bhattacharya, and S. P. Maity, "Comparative study of Radial Basis Function neural network with estimation of Eigenvalue in image using MATLAB," in Recent Advances in Information Technology, ed: Springer, 2014, pp. 141-146.

[7] Y. Wang, M. Chang, H. Chen, and M. Q. Wang, "Application of RBF Neural Network in Intelligent Fault Diagnosis System," in Proceedings of International Conference on Soft Computing Techniques and Engineering Application, pp. 561-566, 2014, 